\title{
On learnability and naturalness as constraints on phonological grammar
}

Hahn Koo and Jennifer Cole

Department of Linguistics, University of Illinois at Urbana Champaign, USA

https://doi.org/10.36505/ExLing-2006/01/0035/000035

\begin{abstract}
We report six experiments on learnability of four non-adjacent phonotactic con-straints which differ in their attested frequency and phonetic conditioning factors; liquid harmony, liquid disharmony, backness harmony, and backness disharmony. Our results suggest that such phonotactic constraints can be implicitly learned from brief experience and that learnability of a phonological grammar may be independ-ent of its attested frequency and phonetic basis.
\end{abstract}

\section{Introduction}

The phonological structures of language are partly shaped by phonetic processes. For example, assimilation is attributed to undershoot and overlap of articulatory gestures (Browman \& Goldstein 1992; Ohala 1990). Learnability is another source of constraint: sound patterns become grammaticized only to the extent that they define robust generalizations over the lexicon that are learnable from the speech environment (Bybee 2001). Thus, we predict that frequently attested phonotactic patterns are those which are both perceptually salient and easily learned. This paper tests this prediction using the experimental technique of artificial grammar learning, where adult subjects learn phonotactic dependencies based on brief exposure to nonce words, as in Onishi et al. (2002).

We compare evidence of learning across four phonotactic constraints involving harmony and disharmony between non-adjacent liquids and high vowels: liquid harmony, liquid disharmony, backness harmony targeting high vowels, backness disharmony targeting high vowels. These constraints have plausible bases in processes of speech production and/or perception, but they differ in their attested frequency in natural languages. Liquid disharmony is more commonly attested than liquid harmony (Hansson 2001), while vowel harmony is more common than vowel disharmony (Pycha et al. 2003). Furthermore, constraints involving non-adjacent vowels are more common than those involving non-adjacent consonants. Our hypothesis is that the lower frequency constraints will also show lesser evidence of learning.

ExLing 2006: Proceedings of 1st Tutorial and Research Workshop on Experimental Linguistics, 28-30 August 2006, Athens, Greece 


\section{Auditory repetition experiments}

We tested learnability of each of four constraints (liquid harmony, liquid disharmony, backness harmony, backness disharmony) in separate experiments (Experiments 1A-1D) through an auditory repetition task. In the study phase, subjects hear and repeat words that instantiate the phonotactic constraint (study words), and in the test phase they hear and repeat new words that are consistent with the constraint (legal words) and words that violate the constraint (illegal words). Mean reaction time measures are compared for legal and illegal words. Evidence of learning was interpreted as significantly shorter mean latencies to legal words than to illegal words.

\section{Methods}

Four experiments investigated learnability of the respective four phonotactic constraints. 15 adult native speakers of English participated in each experiment and received course-credit for compensation.

Stimuli were nonce words of the form $\mathrm{C}_{1} \mathrm{~V}_{1} \cdot \mathrm{C}_{2} \mathrm{~V}_{2} \cdot \mathrm{C}_{3} \mathrm{~V}_{3}$, produced by a male native speaker of English. The first syllable $\left(C_{1} V_{1}\right)$ was either $/ \mathrm{sa} /$ or $/ \mathrm{ke} /$. Elsewhere, consonants and vowels were chosen from $\{s, k, 1, r, a, e, i, u\}$. For each experiment, the words were classified into four types of words: study, legal, illegal, and filler (distracter) words. For example, for the experiment on learnability of backness disharmony, study words and legal words had two high vowels of conflicting backness, illegal words had two high vowels of same backness, and filler words were the remaining words. For each experiment session, 16 study words, 18 legal words, 18 illegal words, and 40 filler words were pseudo-randomly chosen. The chosen words were then distributed across five blocks. Study words recurred in each of five blocks. Legal and illegal words were evenly distributed in the last three blocks. Filler words were evenly distributed in all five blocks.

In each trial, subjects listened to a word through a headset and repeated it as quickly and accurately as possible into a microphone. Latency was measured from the stimulus offset to the response onset for each trial. The entire session was audio-taped for analysis of response accuracy.

\section{Results}

For each subject, latencies were averaged per block and per stimulus type after the following were excluded: (1) errors, (2) responses not detected by the microphone in the first attempt, (3) responses initiated before the word was presented through its penultimate syllable, (4) responses with reaction times of 2.5 standard deviations away from the mean.

For each of the four experiments, a within-subject ANOVA was conducted with block (blocks 3 5) and legality (legal vs. illegal) as factors. The 
effect of legality was significant in experiments on liquid harmony and disharmony, but not in experiments on backness harmony and disharmony. This suggests that subjects learned to generalize the liquid constraints to new instances but failed to learn to generalize the backness constraints. The results of analyses are summarized in Table 1.

Table 1. Results of within-subject ANOVAs.

\begin{tabular}{|l|l|l|}
\hline Constraint (Experiment) & Legality & Legality $\times$ Block \\
\hline Liquid Harmony (1A) & $\mathrm{F}(1,14)=6.278$, & $\mathrm{F}(2,28)=1.487$, \\
\hline Liquid Disharmony (1B) & $\mathrm{F}(1,14)=8.435$, & $\mathrm{F}(2,28)=0.111$, \\
\hline Backness Harmony (1C) & $\mathrm{F}(1,14)=0.164$, & $\mathrm{F}(2,28)=0.289$, \\
\hline Backness Disharmony & $\mathrm{F}(1,14)=0.188$, & $\mathrm{F}(2,28)=0.390$, \\
\hline
\end{tabular}

\section{Grammaticality judgment experiments}

The lack of evidence of learning for the backness constraints may have been task-specific in that repetition facilitation may have been present, but not strong enough to result in significantly shorter repetition latencies with these stimuli. We ran two further experiments to test learnability of liquid harmony (Experiment 2A) and backness harmony (Experiment 2B) with the same nonce word stimuli, but with a grammaticality judgment task in the test phase. After a study phase with an auditory repetition task, subjects were asked to decide for each test word if it belonged to the language exemplified in the study phase (i.e., if it was 'grammatical'). Evidence of learning was interpreted as the subjects' ability to discriminate legal words from illegal words.

\section{Methods}

Subjects in each of the two experiments comprised 15 adult native speakers of English, who received course credit for compensation. The materials were identical to the ones used in the auditory repetition experiments. A session in $2 \mathrm{~A}$ and $2 \mathrm{~B}$ comprised a study phase with three blocks and a test block, and each study block contained the study words and filler words from 1A and 1C, respectively.[j1] The subjects performed the auditory repetition task during the study phase. In the test block, which comprised legal, illegal and filler word, subjects judged for each test word whether it belonged to the language of the study phase by responding "Yes" or "No".

\section{Results}

A $d$ '-score was computed for each subject, where hit-rate was defined as the proportion of "Yes" responses to legal words and false-alarm rate was defined as the proportion of "Yes" responses to illegal words. The log-linear 
rule (Hautus 1995) was applied to account for extreme cases where subjects had a hit-rate of 1.0.

A one-sample $t$-test with $d^{\prime}=0.0$ as the null hypothesis showed that subjects discriminated legal words from illegal words significantly in both the liquid harmony experiment $(\mathrm{t}(14)=3.717, \mathrm{p}=0.002)$ and the backness harmony experiment $(t(14)=3.399, p=0.004)$. The scores between the two experiments were not significantly different $(\mathrm{t}(28)=1.089, \mathrm{p}=0.285)$. Thus, subjects appeared to have learned to generalize backness harmony as well as liquid harmony.

\section{Conclusion}

Our findings from six experiments show that subjects implicitly learned phonotactic constraints from brief experience, and that constraints that differ in attested frequency and phonetic conditioning factors are learned equally well. We conclude that the learnability of a constraint is independent of its phonetic basis and attested frequency in natural languages. However, we also find differences in learning that relate to differences on the experimental task, indicating that future research must consider task factors in relation to phonetic factors in assessing the role of learnability on phonological grammar.

\section{References}

Browman, C. and Goldstein, L. 1992. Articulatory phonology: an overview. Phonetica 49, 155-180.

Bybee, J. 2001. Phonology and Language Use. Cambridge, CUP.

Hansson, G. O. 2001. Theoretical and typological issues in consonant harmony. Doctoral dissertation, Department of Linguistics, University of California at Berkeley.

Hautus, M. 1995. Corrections for extreme proportions and their biasing effects on estimated values of d'. Behavioral Research Methods, Instruments, and Computers $27,46-51$.

Ohala, J. 1990. The phonetics and phonology of aspects of assimilation. In Kingston, E. and Beckman, M. (eds.) 1990, Papers in Laboratory Phonology I: Between the Grammar and the Physics of Speech, 258-275, Cambridge, CUP.

Onishi, K., Chambers, K., and Fisher, C. 2002. Learning phonotactic constraints from brief auditory experience. Cognition 83, B13-B23.

Pycha, A., Nowak, P., Shin, E., and Shosted, S. 2003. In Garding, G. and Tsujimura, M. (eds.) 2003, Proceedings of the XXIIth West Coast Conference on Formal Linguistics, 423-435, Somerville, M.A., U.S.A. 